Als Mittel aus diesen 10 Messungen ergibt sich für $\beta+1$ der Wert 1,62. Die größte gemessene Abweichung von "diesem Mittelwert beträgt $\mathrm{rd}$. $4^{1 / 3} \mathrm{vH}$. Die Genauigkeit einer Einzelmessung dürfte schätzungsweise etwa \pm 3 bis $4 \mathrm{vH}$ betragen. Der theoretische Wert von $\beta+1$ für einen Flügel von dem vorliegenden Achsenverhältnis beträgt 1,71 (vergl. die Zahlentafel auf $S .332$ ). Nach der elementaren Theorie ist, wie wir wissen, $\beta+1=2$. Der experimentelle Wert von $\beta+1$ ist, wie man sieht, um rd. $5,5 \mathrm{vH}$ niedriger als der unter Zugrundelegung der Tragflügeltheorie erhaltene Wert. Die Ursachen für den geringeren experimentellen Wert können folgende sein:

1. Den theoretischen Entwicklungen liegt die Voraussetzung zugrunde, daß die Spannweite des Flügels klein ist gegenüber dem Radius der Flugbahn. Dies war bei den beschriebenen Versuchen nicht in hinreichendem Maße der Fall und es ist vielleicht möglich, daß aus diesem Grunde Abweichungen der beobachteten Art auftreten.

2. Bei dem dicken Flïgel und der verhältnismäßig geringen Geschwindigkeit tritt vermutlich auf der Saugseite Ablösung der Strömung und Totwasserbildung ein. Dieses Totwasser wird durch die Zentrifugalkraft radial nach anßen geschleudert und dadurch auf der Außenhälfte des Flügels die Ablösung 'begünstigt. Verstärkung der Ablösung bedingt Verkleinerung des Auftriebes der äuberen Flügelhälfte und somit kleineres Quermoment. Bei großen Geschwindigkeiten und großen Flügeltiefen (große Reynoldssche Zahlen) tritt erfahrungsgemäß die Totwasserbildung immer mehr in den Hintergrund, so das dann $\beta+1$ möglicherweise dem theoretischen Wert zustreben wird.

Die Versuche bestätigen mit ausreichender Sicherheit das theoretische Resultat, daß nämlich das wirkliche Quermoment kleiner ist als dasjenige, das auf Grund der einfachen geometrischen Vorstellung berechnet wird, bei der der Einfluß des ringförmigen Wirbelbandes auf dem Flügel außer acht gelassen wird.

170

\title{
Über die Spannungen in freitragenden gefüllten Rohren.
}

\author{
Von E. SCHWERIN in Charlottenburg.
}

$\mathrm{I}$ einer 1920 veröffentlichten Arbeit hat Thoma ${ }^{1}$ ) die Spannungen berechnet, die in einem freitragenden, gefüllten Rohr infolge des Gewichts der Flüssigkeit anftreten, und zwar unter der Voranssetzung, daB die Bieguigsfestigkeit der Wandung selbst vernachlässigt werden kann. Hierbei ergab sich, daß die dann allein auftretenden Normal- und Schubspannungen aus den Gleichgewichtsbedingungen gefunden werden können und sich in geschlossener Form angeben lassen.

In der vorliegenden Arbeit soll nun der Einfluß der Biegungs- und"Torsionsfestigkeit der Wandung eingehend verfolgt werden, und zwar nicht nur bei Belastung durch das Flüssigkeits-, sondern auch durch das Eigengewicht des Rohres selbst; ferner wird nicht nur das wagerecht liegende, sondern auch das geneigte Rohr in den Kreis der Betrachtung gezogen werden.

I. Spannungen infolge des Flïsigkeitggewichts im wagerecht liegenden Rohr.

1. Aufstellung der Gleichungen. Das wagerecht

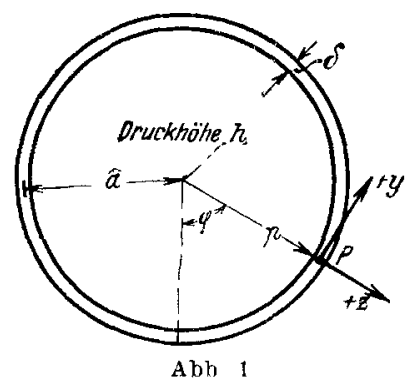
gelagerte Rohr, mit Flïssigkeit vom spez. Gew. $\gamma$ gefüllt, habe die Wandstärke $\delta$, den Radius der Mittelfläche $a$ und die Länge $l$; das Material besitze das spez. Gew. $\gamma_{E}$ und die Elastizitätszahl $E$. In der Zylinderachse sei eine Druckhöhe $h$ vorhanden, so daß nach Abb. 1 der in Richtung des Radius wirkende Flüssigkeitsdruck $p$ in einem Punkt $P$, durch den Zentriwinkel $\varphi$ charakterisiert, beträgt:

$$
p=\gamma(h+a \cos \varphi) \text {. }
$$

Der Pankt $P$ sei zum Anfangspunkt eines Koordinatensystems gewählt, dessen $x$-Achse in Richtung der Rohrachse nach links weist und dessen $y$ - und $z$-Achse wie oben

1) Zeitschr. f. d ges. Turbinenwesen, 1920. 
angegeben gerichtet sind. Auf die nach wachsendem $x$ und $y$ hin gelegenen Seitenflächen des Zylinderelements wirken dann folgende, anf die ganze Wandstärke $\delta$ und die Längeneinheit bezogenen Resultanten von Kräften und Momenten:

länge des Querschnitts des Rohres:

gleichmäBig über die Wandstärke -verteilte Normalspannungen als Zugspannungen positiv, mit der Resultierenden $T_{3}$; linear mit dem Abstand von der Mittelfläche wachsende Biegungsspannungen, statisch gleichwertig einem Kräftepaar rom Moment $G_{1}$, positiv, wenn auf Zubiegung der Schale hinwirkend;

Schubspannungen in Richtung der $z$-Achse mit der Resultierenden $N_{\mathbf{1}}$; Torsionsspannungen statisch gleichwertig einem Torsionsmoment $H_{1}$, positiv, wenn entgegengesetzt dem Uhrzeiger drehend;

Schubspannungen in Richtung der $y$-Achse mit der Resultierenden $S_{1}$;

längs der Zylindererzeugenden:

analoge Spannungsresultanten $T_{2}, N_{3}$ und Spannungsmomente $G_{2}$, gleichfalls als zubiegende Momente positiv;

Schubspannungen in Richtung der negativen $x$-Achse mit der Resultierenden $S_{2}$;

Torsionsspannungen, gleichwertig einem Torsionsmoment $H_{2}$, positiv im Sinne des Uhrzeigers.

Die Formänderung der Schale sei in folgender Weise gekennzeichnet:

$u, v, w$ die in die Richtungen der bezw. $x, y, z$-Achsen fallenden Verschiebungskomponenten von $P$.

$\varepsilon_{1} \varepsilon_{3}$ in der Mittelfluche gemessene Dehnungcn in Richtung der Erzeugenden bezw. senkrecht dazu.

o Winkelverkleinerung des ursprïnglich rechten, von den positiven $x$ - and $y$ Richtungen eingesoblossenen Winkels.

$x_{1} x_{2}$ Krümmungsänderungen in Richtung der Erzeugenden bezw. senkrecht dazu, als Aufbiegungen positiv.

z der gegenseitige Verdrehungswinkel zweier anf derselben Erzengenden im Abstand der Längeneinheit liegender (Querschnittselemente, posiliv im Sinne von $H_{1}$.

Bezeichnet $\sigma$ den Querkontraktionskoelfizienten und wird zur Abkürzung:

$$
D=\frac{E \delta^{3}}{12\left(1-\sigma^{2}\right)}
$$

gesetzt, so sind nach Love1) die Spannungsresultanten bezw. Spannungsmomente mit den Formonderungen in folgender Weise verbunden:

$$
\begin{aligned}
& T_{1}=\frac{12 D}{\delta^{2}}\left(\varepsilon_{1}+\sigma \varepsilon_{2}\right) \\
& T_{2}=\delta^{2} D\left(\varepsilon_{2}+\sigma \varepsilon_{1}\right) \\
& S_{1}=\begin{array}{cc}
12 D & 1-\sigma \omega \\
\delta^{2} & 2
\end{array}
\end{aligned}
$$

$$
\left.\begin{array}{l}
G_{1}=-D\left(x_{1}+\sigma x_{2}\right)+T_{1} \delta^{2} \\
G_{2}=-D\left(x_{2}+\sigma x_{1}\right) \\
H_{1}=D(1-\sigma) \tau-S_{1} \frac{\delta^{2}}{12 a} \\
H_{2}=-D(1-\sigma) \tau
\end{array}\right\}
$$

Die Hinzufügung der $T_{1}$ und $S_{1}$ enthaltenden Korrektionsglieder in den Formeln für $G_{1}$ und $H_{1}$ ist deshalb notwendig, weil die Biegungsverzerrungen $\delta \varkappa, \delta \tau$ klein gegen die Reckungen $\varepsilon$, $\omega$ sind.

Ferner ergeben sich aus den von Love angegebenen allgemeinen Beziehungen, wenn zur Abkürzung:

$$
\xi=\frac{x}{a} \quad \text { व } \xi=U^{\prime} \quad \text { वU }=U^{.}
$$

gescnrieben wird, folgende Formänderungs-Verschiebungsgleichungen:

$$
\left.\begin{array}{ll}
a \varepsilon_{1}=u^{\prime} & a^{2} x_{1}=w^{\prime \prime} \\
a \varepsilon_{2}=v^{\prime}+w & a^{2} x_{2}=w^{\prime}-v^{\prime} \\
a \omega=v^{\prime}+u & a^{2} z=w^{\prime \prime}-v^{\prime}
\end{array}\right\}
$$

1) A. F. H. Love, Lehrb. d. Elastlzitat, deutsch von A. Timpe, Telubner 1907, S. 604, 605, (36) ค. (39). 
während die Gleichgewichtsbedingungen am Zylinderelement lauten:

$$
\begin{array}{lr}
T_{1}{ }^{\prime}-S_{2} \cdot=0 & H_{1}{ }^{\prime}-G_{2}{ }^{\circ}+N_{3} a=0 \\
S_{1}{ }^{\prime}+T_{2}{ }^{\circ}+N_{2}=0 & G_{1}{ }^{\prime}+H_{2} \cdot-N_{1} a=0 \\
N_{1}{ }^{\prime}+N_{2}{ }^{\circ}-T_{2}+a \gamma(h+a \cos \gamma)=0 & -H_{2}+\left(S_{1}+S_{2}\right) a=0
\end{array}
$$

oder auch, wenn $S_{2}$ mit Hilfe der letzten Gleichung und $H_{2}$ mittels (I.) eliminiert wird:

$$
\left.\begin{array}{ll}
T_{1}^{\prime}+S_{1} \cdot\left(1+\frac{\delta^{2}}{12 a^{2}}\right)+{ }_{1}^{\prime}{ }^{\prime}=0 & H_{1}^{\prime}-G_{2}{ }^{\prime}+N_{2} a=0 \\
S_{1}^{\prime}+T_{2}+N_{2}=0 & G_{1}^{\prime}-H_{1} \cdot-S_{1} \cdot a \frac{\delta^{2}}{12 a^{4}}-N_{1} a=0 \\
N_{1}^{\prime}+N_{2} \cdot T_{2}+a \gamma(h+a \cos \varphi)=0 &
\end{array}\right\}
$$

Nun besteht die in diesen Gleichungen aultretende, von den äußeren Kräften abbängige "Störungsfunktion" $a \gamma(h+a \cos q)$ ans den beiden Gliedern $a \gamma h$ und $a^{2} \gamma \cos q$ und es wird daher der schließlich resultierende Gesamtspannungszustand, da für das hier vorliegende lineare Gleichungssystem das Superpositionsgesetz gilt, als Ueberlagerung der den beiden Einflüssen $a \gamma h$ und $a^{2} \gamma \cos \phi$ entsprechenden Spannungszustände erhalten werden können. Man erkennt, daß das konstante Störungsglied $a \gamma h$ einen rotationssymmetrischen, das Glied $a^{2} \gamma \cos \varphi$ hingegen einen in bezug auf $\varphi$ sinusbezw. cosinusförmig verlanfenden Spannungszustand hervorrufen wird, von denen der letztere, also der Fall $h=0$, zunächst betrachtet werde.

2. Der sínusförmige Spannungszustand infolge von $a^{3} \gamma \cos q$. Mit $h=0$ kann wenn zur Abkürzung

$$
\frac{\delta^{2}}{12 a^{2}}=\varepsilon \quad S_{2}=S \quad H_{1}=H
$$

gesetzt wird, den Gleichungen (I), (II), (III) duroh folgenden Ansatz genügt werden:

$$
\begin{aligned}
& T_{1}=\overline{T_{1}} \cos \varphi \quad G_{1}=\overline{G_{1}} \cos \varphi \\
& T_{2}=\overline{T_{2}} \cos \varphi \quad G_{2}=\overline{G_{3}} \cos \varphi \\
& S=\bar{S} \sin \varphi \quad H=\bar{H} \sin \varphi \\
& \varepsilon_{1}=\bar{\varepsilon}_{1} \cos \varphi \quad x_{1}=\bar{x}_{1} \cos \varphi \\
& \varepsilon_{2}=\overline{\varepsilon_{2}} \cos \varphi \quad x_{2}=\overline{x_{2}} \cos \varphi \\
& \infty=\bar{\omega} \sin \varphi \quad \tau=\bar{\tau} \sin \varphi \\
& \overline{N_{1}}=N_{1} \cos \varphi \\
& \overrightarrow{N_{2}}=N_{2} \sin \varphi \\
& u=\vec{u} \cos \varphi \\
& v=\vec{v} \sin \varphi \\
& w=\bar{w} \cos \varphi
\end{aligned}
$$

wo die überstrichenen Größen nur noch Funktionen von $x$ darstellen. Hiermit gehen die Gleichgewichtsbedingungen (III), wenn wir die Querstriche nachträglich fortlassen, über in

$$
\begin{aligned}
& T_{1}^{\prime}+S\left(1^{\prime}+\varepsilon\right)+\frac{H}{a}=0 \\
& S^{\prime \prime}-T_{2}+N_{2}=0 \\
& N_{1}^{\prime}+N_{2}-T_{2}+a^{2} \gamma=0
\end{aligned}
$$

$$
\left.\left.\begin{array}{l}
H^{\prime}+G_{2}+N_{2} a=0 \\
G_{1}{ }^{\prime}-H-S a \varepsilon-N_{1} a=0
\end{array}\right\} . \quad \text { (III } a\right),
$$

während die Formänderungsbedingungen lauten:

$$
\begin{gathered}
a \varepsilon_{1}=u^{\prime}, \quad a \varepsilon_{2}=v+w, \quad a \omega=v^{\prime}-u, \quad a^{2} x_{1}=w^{\prime \prime}, \quad a^{2} x_{2}=-w-v, \quad a^{2} \tau=-w^{\prime}-r \\
T_{1}=\frac{12 D}{\delta^{4}}\left(\varepsilon_{1}+\sigma \varepsilon_{2}\right), \quad T_{2}=\frac{12 D}{\delta^{2}}\left(\varepsilon_{2}+\sigma \varepsilon_{1}\right), \quad S=\frac{12 D}{\delta^{2}} \frac{1-\sigma}{2} \omega \\
G_{1}=-D\left(x_{1}+\sigma x_{2}\right)+T_{1} a \varepsilon, \quad G_{2}=-D\left(x_{2}+\sigma x_{1}\right), \quad H=+D(1-\sigma) \tau-S a \varepsilon .
\end{gathered}
$$

Dieses Gleichungssystem läßt sich nun in folgender Weise $z u$ einer einzigen Gleichung zusammenziehen. Die Addition der mit a multiplizierten ersten Gleichgewichtsbedingung zur letzten ergibt:

$$
a T_{1}^{\prime}+G_{1}^{\prime}+a\left(S-N_{1}\right)=0 \text {. . . . . . . . (3a), }
$$

ferner folgt aus der Subtraktion der zweiten von der dritten Gleichgewichtsbedingung: und zufolge (3a)

$$
N_{1}^{\prime}-S^{\prime}+a^{2} \gamma=0, \text { somit } N_{1}=S-a^{2} \gamma \xi-c_{1}
$$

$$
a T_{1}+G_{1}+a\left(a^{2} \gamma \frac{\xi^{2}}{2}+c_{1} \xi+c_{2}\right)=0
$$

Elimiminiert man aus den Gl. (III a) zunächst $N_{1}$ und $N_{9}$ und zwar mittelst der Beziehungen:

$$
N_{1}=S-a^{2} \gamma \xi-c_{1}, \quad N_{2}=-\frac{1}{a}\left(H^{\prime}+G_{2}\right) \text {. }
$$


so erhalt man folgende drei Gleichgewichtsbedingungen:

$$
\left.\begin{array}{c}
T_{1}^{\prime}+S(1+\varepsilon)+\frac{H}{a}=0, \quad S^{\prime}-T_{2}-\frac{H^{\prime}+Q_{2}}{a}=0, \\
a T_{1}+G_{1}+a\left(a^{2} \gamma \frac{\xi^{2}}{2}+c_{1} \xi+c_{2}\right)=0
\end{array}\right\}
$$

Aus diesen folgt:

$$
\left.\begin{array}{l}
G_{1}=-a T_{1}-a\left(a^{2} \gamma \frac{\xi^{2}}{2}+c_{1} \xi+c_{2}\right) \\
G_{2}=a\left[(2+\varepsilon) S^{\prime}-T_{2}-T_{1}^{\prime \prime}\right], \quad H=-a T_{1}^{\prime}-a S(1+\varepsilon)
\end{array}\right\}
$$

Andererseits liefern die Formänderungsbedingungen (II a) die Beziehungen :

$$
\omega^{\prime}+\varepsilon_{1}=-a\left(x_{2}^{\prime \prime}+x_{1}\right), \quad \varepsilon_{2}=-a x_{2}, \quad \tau=a x_{9}^{\prime} . \quad . \quad \text { (II b). }
$$

Ersetzt man hierin mit Hilfe der Gl. (Ia) die Formänderungen durch die Spannungsresultanten, so erhält man folgende weiteren Gleichungen:

$$
\begin{gathered}
-G_{1}+a \varepsilon T_{1}+\sigma G_{2}+(1+\sigma)\left(H^{\prime}+a S^{\prime} \varepsilon\right)=-a \varepsilon\left[2 S^{\prime}(1+\sigma)+T_{1}-T_{2} \sigma\right] \\
a \varepsilon\left(T_{2}-T_{1}^{\prime} \sigma\right)=G_{2}-G_{1} \sigma+\sigma T_{1} a \varepsilon \\
H+a \varepsilon S+\frac{a \varepsilon}{1+\sigma}\left(T_{2}^{\prime}-T_{1}^{\prime} \sigma\right) \quad 0
\end{gathered}
$$

und schlieblich durch Einführung von (IIIc) in (IIc) drei Gleichungen für $T_{1} T_{2} S$ :

$$
\begin{array}{r}
-(1+\sigma)\left(T_{1}^{\prime \prime}+S^{\prime}\right)+T_{1}(1+\varepsilon)+\sigma S^{\prime}(2+\varepsilon)-\sigma T_{2}+\sigma T_{1}^{\prime \prime} \\
+\varepsilon\left[2 S^{\prime}(1+\sigma)+T_{1}-T_{2} \sigma\right]+a^{2} \gamma \frac{\xi^{2}}{2}+c_{1} \xi+c_{2}=0 . . . \\
\varepsilon\left(T_{2}-T_{1} \sigma\right)-S^{\prime}(2+\varepsilon)+T_{2}-T_{1}^{\prime \prime}-\sigma T_{1}-\sigma\left(a^{2} \gamma \frac{\xi^{2}}{2}+c_{1} \xi+c_{2}\right)=0 . \\
-T_{1}^{\prime}-S^{\prime}+\frac{\varepsilon}{1+\sigma}\left(T_{2}^{\prime}-T_{1}^{\prime} \sigma\right)=0 . . . . . .
\end{array}
$$

Aus der zweiten derselben folgt:

$$
T_{2}=\sigma T_{1}+\frac{1}{1+\varepsilon}\left[S^{\prime \prime}(2+\varepsilon)+T_{1}^{\prime \prime}+\sigma\left(\alpha^{2} \gamma \frac{\xi^{2}}{2}+c_{1} \xi+c_{3}\right)\right.
$$

Setzt man dies in (5) und (7) ein und eliminiert schließlich noch $S$, so erhält man fïr die Längsspannungsresultante $T_{1}$, wenn nur die erste Potenz der sehr kleinen Größe $\varepsilon=\frac{\delta^{2}}{12 a^{2}}$ beibehalten und:

$$
\frac{1-\sigma^{2}}{\varepsilon}=\frac{12 a^{2}}{\delta^{2}}\left(1-\sigma^{2}\right)=v^{4}
$$

gesetzt wird:

wo:

$$
\begin{gathered}
T_{1}(\mathrm{IV})+\alpha_{1} T_{1}^{\prime \prime}+\alpha^{2} T_{1}+\beta_{1} \xi^{2}+\beta_{2} \xi+\beta_{3}=0 \\
\alpha_{1}=-4-2 \varepsilon \frac{2+3 \sigma}{1+\sigma}, \quad \alpha_{2}=v^{4}\left(1+\varepsilon \frac{\sigma^{2}}{1-\sigma^{2}}\right) \\
\beta_{1}=\frac{\gamma}{2} a^{2} \nu^{4} \begin{array}{c}
1+\varepsilon \\
1+3 \varepsilon
\end{array}=\sim \frac{\gamma}{2} a^{2} v^{4}(1-2 \varepsilon), \quad \beta_{2}=c_{1} v^{4} \frac{1+\varepsilon}{1+3 \varepsilon}=\sim c_{1} v^{4}(1-2 \varepsilon) \\
\beta_{3}=c_{2} \nu^{4} \frac{1+\varepsilon}{1+3 \varepsilon}=\sim c_{2} \nu^{4}(1-2 \varepsilon)
\end{gathered}
$$

Das Problem ist somit auf die Integration der Gl. (IV) zurïckgeiührt.

3. Integration. Das vollständige Integral der linearen Differentialgleichang (IV) mit konstanten Koeffizienten lautet:

$T_{1}=C_{1} \sin \left(\mu_{2} \xi\right) \operatorname{Sin}\left(\mu_{1} \xi\right)+C_{2} \sin \left(\mu_{2} \xi\right)\left(\operatorname{Sol}\left(\mu_{1} \xi\right)\right.$

$+C_{3} \cos \left(\mu_{2} \xi\right) \operatorname{Sin}\left(\mu_{1} \xi\right)+C_{4} \cos \left(\mu_{2} \xi\right) \operatorname{Sof}\left(\mu_{1} \xi\right)$
$+\mathfrak{T}_{1_{2}} \xi^{2}+\mathfrak{T}_{1_{1}} \xi+\mathfrak{I}_{1_{0}}$

wo:

$$
\mu_{1}=\sqrt{\frac{\nu^{2}}{2}\left(1-\varepsilon \frac{\sigma^{2}}{1-\sigma^{2}}\right)+1+\varepsilon \frac{2+3 \sigma}{2(1+\sigma)}} \quad \mu_{2}=\sqrt{\frac{\nu^{2}}{2}\left(1-\varepsilon \frac{\sigma^{2}}{1-\sigma^{2}}\right)-1-\varepsilon \frac{2+3 \sigma}{2(1+\sigma)}}
$$

so daß also

$$
\mu_{1}^{2}+\mu_{2}^{2}=v^{2}\left(1-\varepsilon \frac{\sigma^{2}}{1-\sigma^{2}}\right), \quad \mu_{1}^{2}-\mu_{2}^{2}=2+\frac{2+3 \sigma}{1+\sigma}
$$

und ferner: $\mathfrak{I}_{1_{2}}=-\frac{\gamma}{2} a^{2}\left(1-\frac{2-\sigma^{2}}{1-\sigma^{2}} \varepsilon\right), \quad \mathfrak{I}_{1_{1}}=-c_{1}\left(1-\frac{2-\sigma^{2}}{1-\sigma^{2}} \varepsilon\right)$,

$$
\mathfrak{I}_{1_{0}}=-c_{2}\left(1-\frac{2-\sigma^{2}}{1-\sigma^{2}} \varepsilon\right)-\gamma a^{2} \varepsilon \begin{gathered}
2+\sigma \\
1-\sigma^{2}
\end{gathered}
$$

während $C_{1} \ldots C_{4}$ die vier willkürlichen Integrationskonstanten bezeichnen. 
Aus $T_{1}$ lassen sich die übrigen Spannungs- und Formänderungsgrößen ohne Schwierigkeit bestimmen. Man erhält nach $(5 \mathrm{a}),(7 \mathrm{a})$ zunächst fï̈r $S$ :

wo mit der Abkürzung:

$$
\begin{aligned}
S= & S^{(1)} \sin \left(\mu_{2} \xi\right) \operatorname{Sin}\left(\mu_{1} \xi\right)+S^{(2)} \sin \left(\mu_{2} \xi\right)\left(S_{0}\left(\mu_{1} \xi\right)\right. \\
& +S^{(3)} \cos \left(\mu_{2} \xi\right) \operatorname{Sin}\left(\mu_{1} \xi\right)+S^{(4)} \cos \left(\mu_{2} \xi\right)\left(\operatorname{Sof}\left(\mu_{1} \xi\right)\right.
\end{aligned}
$$

$$
\begin{aligned}
& \begin{array}{c}
1-\sigma+\varepsilon^{2}-\sigma^{2} \\
{ }_{2},{ }^{2}
\end{array} \\
& S^{(1)}=C_{2} \mu_{1} \stackrel{\rho_{1}-1}{1-2 \varepsilon}+C_{3} \mu_{2} \stackrel{Q_{1}+1}{1-2} \\
& S^{(3)}=C_{4} \mu_{1} \frac{\varrho_{1}-1}{1-2 \varepsilon}-C_{1} \mu_{3} \frac{\rho_{1}+1}{1-2 \varepsilon}
\end{aligned}
$$

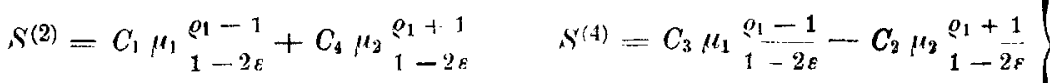

$$
\begin{aligned}
& \widetilde{S}=\left(\gamma r^{2} \xi+r_{1}\right)\left(1-\varepsilon_{1}^{2-\sigma} \sigma_{1-\sigma^{2}}\right)
\end{aligned}
$$

Sodann folgt aus (8) für $T_{2}$, wenn

eingeführt wird:

$$
2+\sigma+\varepsilon \frac{5+16 \sigma+9 \sigma^{2}}{1+\sigma}=\varrho_{9}
$$

$$
\begin{aligned}
T_{2}= & T_{2}^{(1)} \sin \left(\mu_{2} \xi\right) \operatorname{Sin}\left(\mu_{1} \xi\right)+T_{2}^{(2)} \sin \left(\mu_{2} \xi\right)\left(\operatorname{Soj}_{0}\left(\mu_{1} \xi\right)\right. \\
& +T_{2}(3) \cos \left(\mu_{2} \xi\right) \operatorname{Sin}\left(\mu_{1} \xi\right)+T_{2}(4) \cos \left(\mu_{2} \xi\right)(5,0)\left(\mu_{k} \xi\right)
\end{aligned}
$$

wo: $\quad T_{2}^{\prime}(1)=-C_{1} \varrho_{2}+2 C_{4} \mu_{1} \mu_{2}(1+5 \varepsilon), \quad T_{3}(2)=-C_{2} \varrho_{2}+2 C_{3} \mu_{1} \mu_{2}(1+5 \varepsilon)$, und :

$$
T_{2}{ }^{(3)}=-C_{3} \varrho_{2}-2 C_{2} \mu_{1} \mu_{2}(1+5 \varepsilon), \quad T_{2}^{(4)}=-C_{4} \varrho_{2}-2 C_{1} \mu_{1} \mu_{2}(1+5 \varepsilon)
$$

$$
\mathfrak{T}_{2_{2}}=\varepsilon-\frac{\sigma}{1-\sigma^{2}} \gamma_{2}^{a^{2}}, \quad \mathfrak{I}_{2_{1}}=\varepsilon \underset{1-\sigma^{2}}{\sigma} c_{1}, \quad \mathfrak{T}_{\mathfrak{a}_{0}}=\gamma a^{2}+\varepsilon\left(-2 \gamma a^{2} \frac{1+\sigma^{2}}{1-\sigma^{2}}+c_{2} \frac{\sigma}{1-\sigma^{2}}\right) .
$$

Für das Torsionsmoment $H_{1}$ erhalt man nach (III c):

$$
\begin{aligned}
H_{1} & =H_{1}{ }^{(1)} \sin \left(\mu_{2} \xi\right) \operatorname{Sin}\left(\mu_{1} \xi\right)+H_{1}{ }^{(2)} \sin \left(\mu_{2} \xi\right)\left(\operatorname{Soj}\left(\mu_{1} \xi\right)+5\right. \\
& +H_{1}{ }^{\left({ }^{2}\right)} \cos \left(\mu_{2} \xi\right) \operatorname{Sin}\left(\mu_{1} \xi\right)+H_{1}^{(4)} \cos \left(\mu_{2} \xi\right)\left(\mathcal{S}_{0}\left(\mu_{1} \xi\right)\right.
\end{aligned}
$$

wo:

$$
\begin{gathered}
H_{1}^{(1)}=C_{2} \mu_{1}\left[\varrho_{1}-3 \varepsilon\left(1-\varrho_{1}\right)\right]+C_{3} \mu_{2}\left[\varrho_{1}+3 \varepsilon\left(1+\varrho_{1}\right)\right] \\
H_{1}(2)=C_{1} \mu_{1}\left[\varrho_{1}-3 \varepsilon\left(1-\varrho_{1}\right)\right]+C_{4} \mu_{2}\left[\varrho_{1}+3 \varepsilon\left(1+\varrho_{1}\right)\right] \\
H_{1}(3)=C_{4} \mu_{1}\left[\varrho_{1}-3 \varepsilon\left(1-\varrho_{1}\right)\right]-C_{1} \mu_{2}\left[\varrho_{1}+3 \varepsilon\left(1+\varrho_{1}\right)\right] \\
H_{1}(4)=C_{3} \mu_{1}\left[\varrho_{1}-3 \varepsilon\left(1-\varrho_{1}\right]-C_{2} \mu_{2}\left[\varrho^{1}+3 \varepsilon\left(1+\varrho_{1}\right)\right]\right. \\
5=-a\left(\gamma a^{2} \xi+c_{1}\right) \varepsilon \frac{1+2 \sigma}{1+\sigma},
\end{gathered}
$$

während die Spannungsmomente $G_{1} G_{2}$ und die Querkraft $N_{1}$ sich nach (IIIc), (II c), (7) direkt bestimmen aus:

$$
\begin{aligned}
& G_{1}=-a T_{1}-a\left(a^{2} \gamma_{2}^{\xi^{3}}+c_{1} \xi+c^{\natural}\right) \\
& G_{2}=-a \varepsilon T_{2}-\sigma a T_{1}(1+2 \varepsilon)-a \sigma\left(a^{2} \gamma \frac{\xi^{2}}{2}+c_{1} \xi+c^{\natural}\right) \\
& N_{1}=\wedge-a^{2} \gamma \xi-c_{1} .
\end{aligned}
$$

Hiernach werden die Partikulärlösungen:

$$
\begin{aligned}
& G_{1}=\underset{1-\sigma^{2}}{a \varepsilon}\left[-\left(2-\sigma^{2}\right)\left(\gamma \frac{a^{2}}{2} \xi^{2}+c_{1} \xi+c_{2}\right)+\gamma a^{2}(2+\sigma)\right] \\
& G_{2}={ }_{1-\sigma^{2}}\left[-\sigma\left(\gamma \frac{a^{2}}{2} \xi^{2}+c_{1} \xi+c_{2}\right)+\gamma a^{2}(1+2 \sigma)\right] \\
& \vartheta_{1}=-\varepsilon_{1}^{2}-\sigma \\
& 1-\sigma^{2}
\end{aligned}
$$

Für die Querkraft $N_{2}$ folgt nach (IIIa):

$\begin{aligned} \overline{N_{2}} & =N_{2}^{(1)} \sin \left(u_{2} \dot{\xi}\right) \operatorname{Sin}\left(\mu_{1} \xi\right)+N_{2}^{(2)} \sin \left(\mu_{2} \xi\right)\left(\tilde{S}_{0}\left(\mu_{1} \xi\right)\right. \\ & +N_{2}(3) \cos \left(\mu_{2} \xi\right) \operatorname{Sin}\left(\mu_{1} \xi\right)+N_{2}(t) \cos \left(\mu_{2} \xi\right)\left(\Re_{0}\right)\left(\mu_{1} \xi\right)\end{aligned}$ wo mit

$$
\varrho_{3}=1+\varepsilon \frac{3+9 \sigma+5 \sigma^{2}}{1+\sigma},
$$

$$
\begin{aligned}
& N_{2}(1)=-C_{1} \varrho_{3}+6 C_{4} \mu_{1} \mu_{2} \varepsilon, \quad N_{2}(2)=-C_{2} \varrho_{3}+6 C_{3} \mu_{1} \mu_{2} \varepsilon, \\
& N_{2}{ }^{(3)}=-C_{3} \varrho_{3}+6 C_{2} \mu_{1} \mu_{2} \varepsilon, \quad N_{2}^{(4)}=-C_{4} \varrho_{3}-6 C_{1} \mu_{1} \mu_{2} \varepsilon,
\end{aligned}
$$

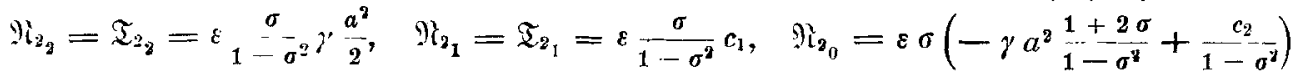


Für die Verschíebungen $u, v, w$ erhält man nach einigen Umformungen:

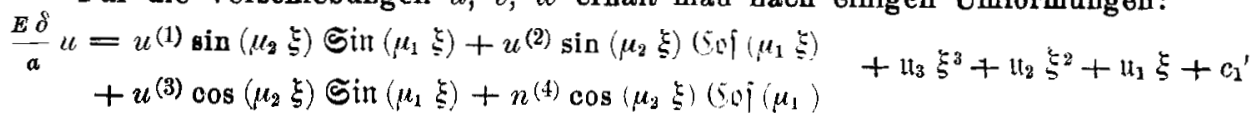

wo, wenn:

$$
\begin{aligned}
& \frac{1-\sigma^{2}}{2^{2}}-\sigma \omega_{1}(2+3 \varepsilon)+\sigma(1+4 t)=\omega^{\prime} \text {, } \\
& \frac{1-\sigma^{2}}{\nu^{2}}-\sigma \varrho_{1}(2+3 \varepsilon)-\sigma(1+4 \varepsilon)=0_{4}^{\prime \prime}, \\
& u^{(1)}=\mu_{1} C_{2} \rho_{4}^{\prime}+\mu_{4} C_{3} \rho_{4}^{\prime \prime}, \quad u^{(2)}=\mu_{1} C_{1} \rho_{4}^{\prime}+\mu_{2} C_{4} \varrho_{4}^{\prime \prime}, \\
& u^{(3)}=\mu_{1} C_{4} \varrho_{4}^{\prime}-\mu_{2} C_{1} \varrho_{4}^{\prime \prime}, \quad u^{(4)}=\mu_{1} C_{3} \varrho_{4}^{\prime}-\mu_{2} C_{2} \varrho_{4}{ }^{\prime \prime}
\end{aligned}
$$

und:

$\mathfrak{u}_{3}=-\gamma \frac{a^{2}}{6}(1-2 \varepsilon), \quad \mathfrak{u}_{3}=-\frac{c_{1}}{2}(1-2 \varepsilon), \quad \mathfrak{u}_{1}=-c_{2}(1-2 \varepsilon)-\gamma a^{2}\left[\sigma+\varepsilon \frac{2-\sigma-2 \sigma^{3}}{1+\sigma}\right]$, während $c_{1}^{\prime}$ eine willkürliche Translationskonstante.

Für $\bar{v}$ folgt der gleiche Ausdruck, nur daß an Stelle $\operatorname{der} u^{(1)}, u^{(2)} \ldots, \mathfrak{H}_{1}, \mathfrak{H}_{2} \ldots$ mit

$$
2+\sigma+2 \varepsilon \frac{1+3 \sigma+\sigma^{2}}{1+\sigma}=\varrho_{5}
$$

die Werte treten:

$$
\begin{gathered}
v_{1}^{(1)}=-C_{1} \varrho_{5}+2 \varepsilon C_{4} \mu_{1} \mu_{2} \frac{3+\sigma}{1+\sigma}, \quad v_{2}^{(2)}=-C_{2} \varrho_{5}+2 \varepsilon C_{3} \mu_{1} \mu_{2} \frac{3+\sigma}{1+\sigma}, \\
v^{(3)}=-C_{3} \varrho_{5}-2 \varepsilon C_{2} \mu_{1} \mu_{2} \frac{3+\sigma}{1+\sigma}, \quad v^{(4)}=-C_{4} \varrho_{5}-2 \varepsilon C_{1} \mu_{1} \mu_{3} \frac{3+\sigma}{1+\sigma^{\prime}} \\
\mathfrak{v}_{4}=\frac{\mathfrak{u}_{3}}{4}, \quad \mathfrak{v}_{3}=\frac{\mathfrak{u}_{2}}{3}, \quad \mathfrak{v}_{2}=\frac{\mathfrak{u}_{1}}{2}+(1+\sigma) \gamma a^{2}\left(1-\varepsilon \frac{2-\sigma}{1-\sigma_{2}^{2}}\right), \\
\mathfrak{v}_{1}=c_{1}^{\prime}+2(1+\sigma)\left(1-\varepsilon \frac{2-\sigma}{1-\sigma^{2}}\right) c_{1} .
\end{gathered}
$$

Auch hier tritt eine weitere, willkürliche Translationskonstante $c_{s}^{\prime}$ auf.

Die radiale Verschiebung $u$ ist endlich gegeben durch:

$$
w=\frac{T_{2}-T_{i} \sigma}{E \delta^{\prime}}-v \text {. }
$$

Von Wichtigkeit ist ferner die Gröbe $w^{\prime}=\frac{d w}{d\left(\frac{x}{a}\right)}$, die die Drehung der Erzengenden bei der Deformation darstellt, Für diese folgt ans (IIa):

und somit nach ( $\mathrm{Ia})$ :

$$
w^{\prime}=-a^{2} \tau-v^{\prime}=-a^{2} \tau-a \omega-u
$$

$$
w^{\prime}=-\frac{a^{2}}{D(1-\sigma)}(H+3 a \varepsilon S)-u
$$

Durch dio im vorstehenden angegebenen Werte sämtlicher Spannungs- und Deformationsgrößen ist der Spannungszustand vollstundig und eindeutig bestimmt und es läbt sich jetzt folgendes orkennen:

Die $C_{1} \ldots \ldots C_{4}$ enthaltenden Teile der Lösungen, die die Integrale des homogenen Gleichungasystems darstellen, nehmen von den Rohrenden aus rasch ab, sind jedoch für die Spannungsverteilung an den letzteren bezw. den Versteifungsringen von Bedeutung. Gegen die Mitte jeder Rohröffnung hin werden jedoch die durch die deutsehon Buchstaben gekennzeichneten Partikulärlösungen fast allein mafgebend sein. Nun liefern die letzteren für die Biegungsmomente $G_{1} G_{2} H$, sowie für $N_{1} N_{2}$ Werte von der GröBen. ordnung $\varepsilon a T$ bezw. $\varepsilon T$, d. h. da $\varepsilon=\frac{\delta^{2}}{12 a^{2}}$ meist sehr klein, ist der Linfluß der Biegungssteifigkeit der Rohrwandung selbst sehr gering.

Noch deullicher wird dies, wenn man den Quotienten $\frac{\mathfrak{S}}{\mathfrak{T}}$ bildet, der das Herausrücken der Stützlinie aus der Mittelfläche angibt. Man erhält z. B. in der Mitte eines an seinen beiden Enden frei gestuitzten Rohres, wenn

$$
\mathfrak{I}_{1(0)}=\gamma \frac{l^{2}}{8}, \quad \mathfrak{I}_{(0)}=\gamma a^{2}
$$


die Längsspannungen ohne Berücksichtigung der Biegungsfestigkeit bezeichnen:

$$
\frac{S_{1}}{\mathfrak{T}_{1(0)}}=\delta \frac{\delta}{12 a}\left(\frac{2-\sigma^{2}}{1-\sigma^{2}}+8 \frac{a^{2}}{l^{2}} \frac{2+\sigma}{1-\sigma^{2}}\right) ; \quad \frac{B_{2}}{\mathfrak{T}_{2(0)}}=\delta \frac{\delta}{12 a}\left(\frac{1+2 \sigma+\sigma^{3} \frac{l^{2}}{8 a^{2}}}{1-a^{2}}\right)
$$

d. h. Pür das schon verbältnismäBig dickwandige Rohr von $\frac{\delta}{a}=\frac{1}{20}$ wird bei $\frac{a}{l}=\frac{1}{5}$, $\sigma=0,3:$

$$
\frac{B_{1}}{\mathfrak{T}_{1}(0)}=\sim \frac{1}{80} \delta=\frac{\dot{B}_{1}}{\mathfrak{L}_{2(0)}}
$$

Es ist jedoch bemerkenswert, daß mit wachsender Rohrlänge unter sonst gleichen Umsiänden der Einfluß $\mathbb{B}$ von $\mathbb{S}_{2}$ zu-, der von $\mathbb{B}_{1}$ hingegen abnimmt.

4. Auilagerbedingungen. Die für die Bestimmung der 8 Konstanten $C_{1} C_{2} C_{3}$ $C_{4} c_{1} c_{2} c_{1}^{\prime} c_{2}^{\prime}$ maßgebenden Randbedingungen lauten für die praktisch rorkommenden Stützungen eines Rohrendquerschnitts wie folgt:

In einem vollkommen eingespannten Endquerschnitt mus sein:

$$
u=0 \quad v=0 \quad w=0 \quad w^{\prime}=\frac{d w}{d\left(\frac{x}{a}\right)}=0 .
$$

Ist hingegen die Stützung derart, daß das Rohr durch einen starren Deckel abgeschlossen ist, der nur vertikal gestützt ist, jedoch sich nngebindert in der Längsrichtnng verschieben und sich auch neigen kann, so lauten bei gelenkigem Anschluß des Rohrs an den Deckel die Bedingungen im Endquerschnitt:

$$
G_{1}=0 \quad T_{1}=0 \quad w=0 \quad v=0
$$

hingegen bei biegrungsfestem Anschluß des Rohrs an den Deckel:

$$
G_{1}+a T_{1}=0 \quad w^{\prime}=0 \quad w=0 \quad v=0 .
$$

Eine besondere Betrachtung eriordert der Fall des kontinuierlichen Rohrstranges, der mittels starrer Versteifungsringe unsenkbar gelagert ist. Bei diesem sind an jeder Mittelstuitze folgende Bedingungen zu befriedigen, wenn die an derselben zusammenstoßenden Oeffnungen den Index $m$ bezw. $m+1$ ebenso wie die zugehörigen Spannungsund DeformationsgröBen erhalten:

$$
\begin{aligned}
& T_{1(m)}=T_{1(m+1)} \quad u_{(m)}=u_{(m+1)}, \quad v_{(m)}=0 \quad w(m)=0 \\
& G_{1(m)}=G_{1(m+1)} \quad w_{(m)}=u_{(m+1)}^{\prime} \quad \boldsymbol{v}_{(m+1)}=0 \quad w_{(m+1)}=0
\end{aligned}
$$

Man erhält also an jeder Mittelstïtze 8, an jeder Endstütze 4, im ganzen also bei $n$ Oeffnungen: $8(n-1)+2.4=8 n$ Gleichungen, denen in jeder Oeffnung $8 \mathrm{zu}$ bestimmende Konstanten, im ganzen also $8 n$ Unbekannte gegienüberstehen; die Zahl der letzteren reduziert sich jedoch bei Symmetrie auf die Hälfte.

5. Der rotationssymmetrische Spannungszustand infolge von $h \gamma$. $\mathrm{Zu}$ den in 2 bis 4 berechneten Spannungen sind nun nach 1 noch die sich infolge des Störungsgliedes $\gamma h$ erge benden Spannungen zu überlagern. Da dieselben in bezug auf die Rohrachse gleichmäßig verteilt sind, fallen in den Gl. (I), (II), (III) alle Ableitangen nach $p$ fort; ebenso verschwinden auch $N_{2} H S \omega \tau v_{y}$ Wir erhalten daher in diesem Falle folgendes Gleichungssystem:

$$
\begin{array}{cc}
T_{1}=\frac{12 D}{\delta^{2}}\left(\varepsilon_{1}+\sigma \varepsilon_{2}\right) & G_{1}=-D\left(x_{1}+\sigma x_{2}\right)+T_{1} a \varepsilon \\
T_{2}=\frac{12 D}{d^{2}}\left(\varepsilon_{2}+\sigma \varepsilon_{1}\right) & G_{3}=-D\left(x_{2}+\sigma x_{1}\right) \\
a \varepsilon_{1}=u^{\prime}, a \varepsilon_{2}=u^{\prime} ; & a^{2} x_{1}=w^{\prime \prime}, x_{2}=0 . \quad . \\
T_{1}^{\prime}=0, \text { somit } T_{1}=c_{1} \quad N_{1}^{\prime}-T_{2}+a \gamma h=0, \quad \sigma_{1}^{\prime}-N_{1} a=0 .
\end{array} .
$$

Mit Rücksicht auf $x_{2}=0$ (nach IId) folgt für Gl. (Id):

$$
\begin{array}{ll}
T_{1}=\frac{12 D}{d^{2}}\left(\varepsilon_{1}+\sigma \varepsilon_{2}\right)=c & G_{1}=-D \varkappa_{1}+T_{1} a \varepsilon=-D x_{1}+c a \varepsilon \\
T_{2}=\frac{12 D}{\delta^{2}}\left(\varepsilon_{2}+\sigma \varepsilon_{1}\right) & G_{2}=-D \sigma \varkappa_{1} .
\end{array}
$$
sammenziehen.

Dieses Gleichungssystem läbt sich in folgender Weise auf eine Gleichung zu- 
Die Elimination von $N_{1}$ aus (III) ergibt:

hierzu mit $T_{1}=c$ nnd (IId)

$$
\begin{aligned}
& G_{1}{ }^{\prime \prime}-a T_{2}+a^{2} \gamma h=0 \quad \text {. . . . . . . . (IIIc), } \\
& \varepsilon_{2}{ }^{\prime \prime}=a x_{1} ; \quad x_{2}=0 \text {. . . . . . . . . (II c). }
\end{aligned}
$$

Ersetzt man in (IIc) Dehnungen und Krümmungsänderungen mittels (Id), so erhält man:

mithin :

$$
\varepsilon_{2}^{\prime \prime}=\frac{T_{2}^{\prime \prime}-T_{1}^{\prime \prime} \sigma}{E \delta}=\frac{G_{1}(\mathrm{IV})}{E \delta a}=a \varkappa_{1}=\frac{a}{D}\left(c a \varepsilon-G_{1}\right),
$$

$$
G_{1}^{(I V)}+\frac{12 a^{2}}{d^{2}}\left(1-\sigma^{2}\right) G_{1}-c a\left(1-\sigma^{2}\right)=0 .
$$

Das vollstandige Integral dieser Gleichung lautet:

$$
G_{1}=C_{1} \sin (\mu \xi) \Subset \operatorname{in}(\mu \xi)+C_{2} \sin (\mu \xi)(\mathfrak{p}\{(\mu \xi)
$$

wobei

$$
+C_{3} \cos (\mu \xi) \operatorname{Sin}(\mu \xi)+C_{4} \cos (\mu \xi)\left(S_{0}\{(\mu \xi)+c a \varepsilon\right.
$$

$$
\mu=\frac{\nu}{\sqrt{2}}, \quad \frac{12 a^{2}}{\delta^{2}}\left(1-\sigma^{2}\right)=\nu^{4}
$$

Nach Bestimmung von $G_{1}$ folgt für $T_{2}$ nach (IIIc):

$$
\begin{aligned}
T_{2} & =G_{1}^{\prime \prime}+a \gamma h \\
& =\frac{n^{2}}{a}\left[-C_{4} \sin (\mu \xi) \operatorname{Sin}(\mu \xi)-C_{3} \sin (\mu \xi)\left(50 j(\mu \xi)+C_{z} \cos (\mu \xi) \operatorname{Sin}(\mu \xi)+C_{1} \cos (\mu \xi)(501(\mu \xi)],\right.\right. \\
& +a \gamma h
\end{aligned}
$$

ferner für $G_{2}$ nach $(\mathrm{Id})$ :

$G_{2}=\sigma G_{1}-\sigma c a \varepsilon$

$=\sigma\left[C_{1} \sin (\mu \xi) \sin (\mu \xi)+C_{2} \sin (\mu \xi)\left(5_{0} i(\mu \xi)+C_{3} \cos (\mu \xi) \operatorname{Sin}(\mu \xi)+C_{4} \cos (\mu \xi)(5 \mathfrak{D}\{(\mu \xi)]\right.\right.$.

Für die Querkraft $N_{1}$ liefert die letzte der Gleichgewichtsbedingungen (IIId):

$N_{1}=\frac{k^{\prime}}{a V^{\prime}}\left[\left(C_{2}-C_{3}\right) \sin (\mu \xi) \operatorname{Sin}(\mu \xi)+\left(C_{1}-C_{4}\right) \sin (\mu \xi) \operatorname{Soj}(\mu \xi)+\left(C_{1}+C_{4}\right) \cos (\mu \xi) \operatorname{Sin}(\mu \xi)\right.$

während man für die Verschiebungen erbält:

$$
+\left(C_{2}+C_{3}\right) \cos (\mu \xi)(5,0 ;(\mu \xi)]
$$

$$
\begin{gathered}
w=a \varepsilon_{2}=\frac{a}{\dot{E} \delta}\left(T_{2}-T_{1} \sigma\right) \\
u=a \int \varepsilon_{1} d \xi=\frac{a}{E \delta} \int\left(T_{1}-T_{2} \sigma\right) d \xi=\frac{a}{E \delta^{\delta}} \int\left\{c-\sigma\left(N_{1}^{\prime}+\gamma a h\right)\right\} d \xi \\
=-\frac{a \sigma}{E \delta} N_{1}+\frac{a}{E \delta}(c-\sigma \dot{a} \gamma h) \xi+c^{\prime}\left(c^{\prime}=\text { willk. Konst. }\right)
\end{gathered}
$$

und die Tangentendrehung $w^{\prime}$ sich ergibt zu:

$$
\begin{aligned}
w^{\prime} & =\frac{a}{E \delta^{\prime}} T_{2}^{\prime} \\
& =\frac{\nu^{3}}{\varepsilon \delta \sqrt{2}}\left[\begin{array}{l}
-\left(C_{2}+C_{3}\right) \sin (\mu \xi) \operatorname{Sin}(\mu \xi)-\left(C_{1}+C_{4}\right) \sin (\mu \xi)\left(S_{01}(\mu \xi)\right. \\
+\left(C_{1}-C_{4}\right) \cos (\mu \xi) \operatorname{Sin}(\mu \xi)+\left(C_{2}-C_{3}\right) \cos (\mu \xi) \operatorname{Sof}(\mu \xi)
\end{array}\right]
\end{aligned}
$$

Hiermit sind sämtliche Spannungen und Formänderungen bestimmt, sobald noch die Werte der 6 Konstanten $\left.C_{1} C_{2} C_{3} C_{4} C c^{1}\right)$ aus den im folgenden angegebenen Randbedingungen ermittelt sind.

Die Bedingungen, denen die Spannungen und Formänderungen in einem Endquerschnitt genügen müissen, sind die folgenden:

In einem eingespannten Endquerschnitt mufs sein:

$$
u=0 \quad w=0 \quad w^{\prime}=0 \text {. }
$$

Bei Abschluß durch starre, nur vertikal gestützte, drehbare, in der Längsrichtung frei verschiebliche Deckel hat man, wenn das Rohrende gelenkig an den Deckel angeschlossen ist:

$$
G_{1}=0 \quad T_{1}=0 \quad w=0,
$$

während bei biegungsfestem Anschluß des Rohres an den Deckel die Bedingungen lauten:

$$
w^{\prime}=0 \quad T_{\mathfrak{s}}=0 \quad w=0 .
$$

1) Diese sind natarlich nieht identiseh mit den in Losungen unter 2. anftretenden Konstanten. 
Bei kontinuierlichen Rohrsträngen sind über jeder Mittelstütze folgende 6 Bedingungen zu erfüllen:

$$
\begin{array}{lll}
T_{1(m)}=T_{1(m+1)} & u(m)=u(m+1) & w_{m}=0 \\
G_{1}(m)=G_{1}(m+1) & w^{\prime}(m)=w^{\prime}(m+1) & w_{m+1}=0,
\end{array}
$$

so daB man also bei $n$ Oeffnungen: $6(n-1)+2 \cdot 3=6 n$ Gleichungen für die $6 n$ Konstanten - bei Symmetrie nur die Hälfte - erhält.

Nach Bestimmung der Konstanten und Einsetzung derselben in die obigen Spannungen und Deformationen können die unter 2. und 3 . bestimmten Spannungszustände überlagert und so die Gesamtspannungen infolge des Flüssigkeitsgewichtes und Uoberdruckes erhalten werden, wobei bemerkt sei, daB, wenn kein Ueberdruck vorhanden, in den Formeln unter 3. $h=a$ einzusetzen ist.

\section{Spannungen infolge des Rohreigengewichts im wagerecht liegenden Rohr.}

Die Spannnngen des Rohreigengewichts , Eelbst werden im allgemeinen gegenüber denen des Flüssigkeitsgewichts und Ueberdrucks zurücktreten.

Immerhin besitzt z. B. ein Rohrstück von $1 \mathrm{~cm}$ Lănge bei einer Wandatärke $\delta$ einem Radius $a$, spez. Gew. $\gamma_{E}$ ein Gewicht $2 \pi a \delta \gamma_{E}$, die eingeschlossene Flüssigkeit vom spez. Gew. $\gamma_{F^{\prime}}$ hingegen ein solches von $\pi a^{2} \gamma_{F}$. Die Gewichte verhalten sich also wie

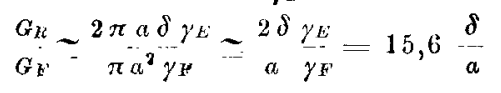

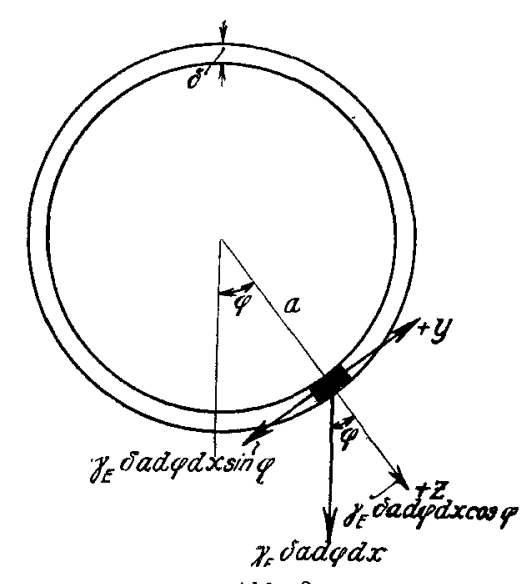

Abb. 2

für ein eisernes $\left(\gamma_{L_{i}}=7,8 \mathrm{t} / \mathrm{m}^{3}\right)$ Rohr mit Wasserfïllung $\left(\gamma_{F^{\prime}}=1 \mathrm{t} / \mathrm{m}^{3}\right)$.

Für ${ }_{a}^{d}={ }_{40}^{1}$ würde hiernach das Rohrgewicht etwa $39 \mathrm{vH}$ des Flïssigkeitsgewichts und für $\frac{\delta}{a}=\frac{1}{100}$ noch etwa $16 \mathrm{vH}$ des letzteren betragen. Es sind also infolge des Rohrgewichts immerhin nicht ganz unbeträchtliche Spannungen zu erwarten, die wir im folgenden untersuchen wollen.

1. Aufstellung der allgemeinen Gleichun-

gen. Aus einem wagerecht liegenden Rohr von der Wandstärke $\delta$, dem Radius $a$, dem spez. Gew. $\gamma_{E}$ sei ein unendlich schmaler Ring von der Breite $d x$ herausgeschnitten. (Abb. 2.)

Dann besitzt ein Bogenelement desselben von der Länge $a d \varphi$ das Gewicht $\gamma_{E} \delta a d \varphi d x$, dessen Komponenten in der $Y$-bezw. Z-Richtung sind:

wenn $\gamma_{E} \frac{\delta}{a}=\gamma_{e}^{\prime}$ gesetzt wird.

$Y=-\gamma_{E} \delta a d \eta d x \sin \varphi=-\gamma_{x^{\prime}} a^{2} d \varphi d x \sin \varphi$

$Z=+\gamma_{E} \delta a d \varphi d x \cos \varphi=+\gamma_{E^{\prime}}^{\prime} a^{2} d \varphi d x \cos \varphi$,

Nach S. 342 lauten daher die Gleiehgewichtsbedingungen:

$$
\left.\begin{array}{lc}
T_{1}^{\prime}-S_{2}=0 & H_{1}^{\prime}-G_{2}+N_{2} a=0 \\
S_{1}^{\prime}+T_{y}+N_{2}-a^{2} \gamma_{E}^{\prime} \sin \varphi=0 & G_{1}^{\prime}+H_{2}-N_{1} a=0 \\
N_{1}^{\prime}+N_{3}-T_{2}+a^{2} \gamma_{E}^{\prime} \cos \varphi=0 & -H_{2}+\left(S_{1}+S_{2}\right) a=0
\end{array}\right\}
$$

während die Formänderungsgleichungen (I) (II) unverändert bleiben.

Man überzengt sich nun leicht, daß die obigen Gleichungen ebenfalls durch den sinus- bezw. cosinusförmigen Ansatz befriedigt werden können und daß dann die Gleichgewichtsbedingungen die Form annehmen $\left.{ }^{1}\right)$ :

$$
\begin{array}{ll}
T_{1}+S(1+\varepsilon)+\stackrel{B}{a}=0 & \\
S^{\prime}-T_{2}+N_{2}-a^{2} \gamma_{E}^{\prime}=0 & H_{1}^{\prime}+G_{2}+N_{2} a=0 \\
N_{1}^{\prime}+N_{2}-T_{1}+a^{2} \gamma_{E^{\prime}}^{\prime}=0 & G_{1}^{\prime}-H-S^{\prime} a \varepsilon-N_{1} a=0
\end{array}
$$

während die Formänderungen sich wieder nach (Ia) (II a) bestimmen.

1) Wiederum unter Fortlassung der Striche über den Ruchstaben. 
Im folgenden soll nun der Einfluß des in der zweiten Gleichgewichtsbedingung auftretenden Störungsgliedes getrennt von dem der dritten Gleichung verfolgt werden.

2. Diskussion der Spannungen. a) Einfluß des Störungsgliedes $+a^{2} \gamma_{E^{\prime}}$ der dritten Gleichgewichtsbedingung. Wenn, wie wir jetzt zunächst annehmen, das Störungsglied $-a^{2} \gamma_{E}^{\prime}$ in der $Y$-Richtung nicht vorhanden ist und nur in der $Z$-Richtung eia solches von der Größe $+a^{2} \gamma_{E^{\prime}}$, so stimmen die Gl. (IIIf) vollkommen mit den Gl. (IIIa) überain, wenn in den letzteren statt $\gamma$ das reduzierte spez. Gew. des Rohrmaterials $\gamma_{E}{ }^{\prime}=\gamma_{E} \frac{\delta}{a}$ eingesetzt wird. Hieraus geht hervor, daß das Störungsglied in der Z-Richtung $+a^{2} \gamma_{E}^{\prime}$ Spannungen hervorruft, die aus den im I. Abschnitt angegebenen Spannungen durch Multiplikation mit dem Verhältnis $\frac{\gamma E^{\prime}}{\gamma}$ abgeleitet werden können. Die Konstanten $C_{1} \ldots C_{4} c_{1} c_{2} c_{1}^{\prime} c_{3}^{\prime}$ verkleinern sich also im Verhältnis $\frac{\gamma E^{\prime}}{\gamma}$ und in den Partikularlösungen ist stat $\gamma_{E}^{\prime}$ statt $\gamma$ einzusetzen.

b) Einfluß des Störungsgliedes - $a^{2} \gamma_{z^{\prime}}$ der zweiten Gloichgewichtsbedingnng. Dieser EinfluB ist zwar nicht mehr so einfach zu übersehen, wie der des Gliedes in der $Z$-Riohtung. Nimmt man jedoch mit den Gl. (IIIf) sowie den Formänderungsgleichungen ( Ia) (II a) die gleichen Transformationen vor, die S. 342 bis 343 zur Aufstellung der Hauptgleichung für $T_{1}$ gefïhrt haben, so ergibt sich fïr $\bar{T}_{1}$ genau dieselbe Gl. (IV) wie oben, so daß also das Störungsglied $-a^{2} \gamma_{E}^{\prime}$ der $Y$-Richtung dem Sinne und der Größe nach genau die gleichen Spannungen $\overline{T_{1}}$ hervorruft wie $+a^{2} \gamma_{E^{\prime}}$ der $Z$ Richtung. Dasselbe ergibt sich für die Größen $\bar{S}_{1} G_{1} \bar{N}_{1} \bar{H}_{1}$ da die Gleichungen, aus denen sie nach Bestimmung von $\bar{T}_{1}$ gefunden werden, dieselben bleiben.

Anders verhălt es sich jedoch mit den Größen $\overline{T_{2}} \overline{N_{2}} \overline{G_{2}}, \overline{u_{1}} \bar{v} \bar{w}$ ) für die zwar die homogenen Lösungen dieselben bleiben, die in deutschen Buchstaben geschriebenen Lösungen jedoch folgende Werte annnehmen ${ }^{1}$ ):

$$
\begin{aligned}
& \tau_{y x^{\prime}}=-\gamma^{\prime} x^{\prime} \frac{a^{2}}{1+\varepsilon}+{ }_{1-\sigma^{2}}^{\varepsilon \sigma}\left(\begin{array}{c}
\gamma^{\prime} \\
2
\end{array} a^{2} \xi^{2}+c_{1}^{*}+c_{2}^{*}\right)+\gamma_{E}^{\prime} a^{2}\left(1-2 \varepsilon \frac{1+\sigma^{2}}{1-\sigma^{2}}\right)
\end{aligned}
$$

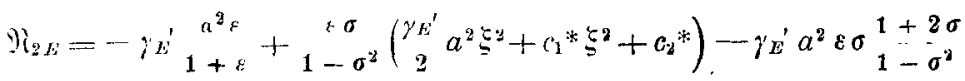

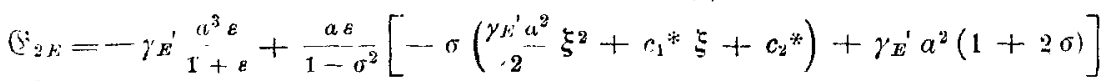

$$
\begin{aligned}
& { }_{a} \delta \mathrm{1l}_{E}=\mathrm{d}_{3 E} \xi^{3}+\mathfrak{l l}_{2} \xi^{2} \mathfrak{1 1}_{1 E} \xi+c_{1} \\
& =\sigma \gamma^{\prime}{ }^{\prime} \frac{a^{2} \xi}{1+\xi}-(1-2 \varepsilon)\left(\gamma_{E^{\prime}} u_{6}^{2} \xi^{3}+{ }_{2}^{c_{1}^{*}} \xi^{2}+r_{z}^{*} \xi\right)-\gamma_{x^{\prime}} a^{2} \xi\left[\sigma+\varepsilon \frac{2-\sigma-2 \sigma^{3}}{1+\sigma}\right]+c_{1}^{* *} \\
& \frac{E \delta}{a} \mathrm{r}_{E}=\mathfrak{r}_{4 E} \xi^{4}+\mathfrak{v}_{3 E} \xi^{3}+\mathfrak{r}_{2 E} \xi^{2}+\mathfrak{v}_{1 E} \xi+c_{2}^{*} \\
& =\sigma \gamma_{F}^{\prime} a_{2(1+\varepsilon)}^{a^{2} \xi^{2}}-(1-2 \varepsilon)\left(\gamma^{\prime} k^{\prime} a^{2} \xi^{4}+\frac{c^{*} \xi^{3}}{6}+\frac{c^{2 *} \xi^{2}}{2}\right) \\
& -\gamma a^{2} \xi^{2}\left[-1-\frac{\sigma}{2}+\varepsilon\left(\frac{2-\sigma}{1-\sigma}+\frac{2-\sigma-\sigma^{3}}{2(1+\sigma)}\right)\right]+2(1+\sigma)\left(1-\varepsilon \frac{2-\sigma}{1-\sigma^{2}}\right) c_{1}^{*} \xi+c_{1} * \xi+c_{2}{ }^{*} * \\
& { }_{a}^{E \delta} \mathfrak{w}_{E}=\mathfrak{T}_{2 k}-\mathfrak{S}_{1}-\mathbf{r}_{E} \frac{E \delta}{a} \text {. }
\end{aligned}
$$

c) Gesamtspannungen und Formänderungen infolge des Rohrgewichts. Nach vorstehendem lassen sich nun die Gesamtspannungen und Formänderungen infolge des Rohrgewichts, die durch den Index y gekennzeichnet seien, durch einfache Superposition der den beiden Störungsgliedern entsprechenden Spannungszustände ermitteln. Man findet auf diesem Wege folgende Werte:

$$
\mathfrak{I}_{1 !}=\mathfrak{I}_{1_{2}} \xi^{2}+\mathfrak{I}_{1_{1}} \xi+\mathfrak{I}_{1_{0}}
$$

$$
\begin{array}{r}
\text { wo } \\
\mathfrak{I}_{1,2}=-\gamma^{\prime} \dot{k}^{\prime} a^{2}\left(1-\frac{2-\sigma^{2}}{1-\sigma^{2}} \varepsilon\right), \quad \mathfrak{I}_{1_{1}}=-c_{\mathrm{s} g}\left(1-\frac{2-\sigma^{2}}{1-\sigma^{2}} \varepsilon\right), \\
\mathfrak{I}_{1_{0}}=-c_{2 g}\left(1-\frac{2-\sigma^{2}}{1-\sigma^{2}} \varepsilon\right)-2 \gamma_{E^{\prime}} a^{2} \varepsilon \frac{2+\sigma}{1-\sigma^{2}} ;
\end{array}
$$

1) Der Index $E$ soll andeuten, daB es sich um Teillosungen infolge kigengewloht des Rohres handelt. 
$\mathfrak{S}_{1,}=\left(2 \gamma_{E}^{\prime} a^{9} \xi+c_{3 y}\right)\left(1-\varepsilon \frac{2-\sigma}{1-\sigma^{2}}\right), \quad \mathfrak{I}_{2 g}=\mathfrak{I}_{2_{2}} \xi^{2}+\mathfrak{I}_{2:} \xi+\mathfrak{I}_{2_{0}}$

wo

$\mathfrak{I}_{2_{2}}=\varepsilon \frac{\sigma}{1-\sigma^{2}} \gamma_{E}^{\prime} a^{2}, \quad \mathfrak{I}_{2_{1}}=\frac{\varepsilon \sigma}{1-\sigma^{2}} c_{1 g}, \quad \mathfrak{I}_{2_{0}}=\gamma_{E^{\prime}} a^{2}+\varepsilon\left(-\gamma_{E^{\prime}}^{\prime} a^{2} \frac{1+3 \sigma^{3}}{1-\sigma^{2}}+c_{2 g}-{ }_{1-\sigma^{2}}\right)$,

$\left(G_{1 \eta}=\frac{a \varepsilon}{1-\sigma^{2}}\left[-\left(2-\sigma^{2}\right)\left(\gamma_{E^{\prime}}^{\prime} a^{2} \xi^{2}+c_{1 y} \xi+c_{2 g}\right)+2 \gamma^{\prime} a^{2}(2+\sigma)\right]\right.$

$\mathfrak{G}_{2 g}=\underset{1-\sigma^{2}}{a \varepsilon}\left[-\sigma\left(\gamma_{E}^{\prime} a^{2} \xi^{2}+c_{1 g} \xi+c_{2 g}\right)+\gamma^{\prime} a^{\prime}\left(1+4 \sigma+\sigma^{2}\right)\right]$

$\xi_{g}=-a\left(2 \gamma_{E}^{\prime} a^{2} \xi+c_{1 g}\right) \varepsilon \frac{1+2 \sigma}{1+\sigma}$

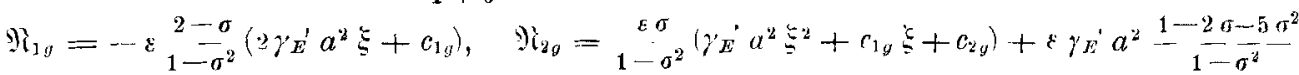

und schließlich für die Verschiebungen:

$$
\begin{aligned}
& { }_{a}^{E \delta} \mathrm{1t}_{g}=-(1-2 \varepsilon)\left(\gamma^{\prime} \frac{a}{3}_{3}^{2} \xi{ }^{3}+\frac{c_{1 g} \xi^{2}}{2}+c_{z g} \xi\right)-\gamma_{E^{\prime}} a^{2} \xi\left[\sigma(1+\varepsilon)+2 \varepsilon \frac{2-\sigma-\sigma^{3}}{1+\sigma}\right] \\
& \underset{a}{E \delta} \mathfrak{b}_{g}-\mathfrak{v}_{4 g} \xi^{4}+\mathfrak{y}_{8} g \xi^{3}+\mathfrak{v}_{2 g} \xi^{2}+\mathfrak{v}_{g} \xi+c_{g}^{\prime} \xi+c_{2 g},
\end{aligned}
$$

wo

$$
\begin{aligned}
& \mathrm{v}_{4 g}=\frac{-\gamma E^{\prime} a^{2}}{12}-(1-2 \varepsilon), \quad \mathrm{v}_{3 g}=-\frac{-\epsilon_{1 g}}{6}(1-2 \varepsilon) \\
& \mathrm{v}_{2 g}=\frac{-c_{2 g}}{2}(1-2 x)+\frac{\gamma F^{\prime} \mathrm{a}^{2}}{2}\left[4+3 \sigma+\varepsilon \frac{-12+\sigma+2 \sigma^{2}+3 \sigma^{3}-2 \sigma^{4}}{1-\sigma^{2}}\right] \\
& n_{1 g}=2(1+\sigma)\left(1-\varepsilon \frac{2-\sigma}{1-\sigma^{2}}\right) c_{1 !}
\end{aligned}
$$

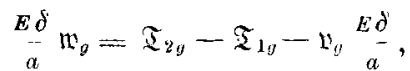

sowie für die Tangentendrehung (vergl. S. 345)

$$
{ }_{a}^{E \delta} \mathfrak{w}^{\prime}=-\frac{12 a(1+\sigma)}{\delta^{2}} 5_{g}-3(1+\sigma) \Im_{g}-\mathfrak{u}_{g} \frac{E \delta}{a},
$$

whhrend die homogenen Lösungen formal

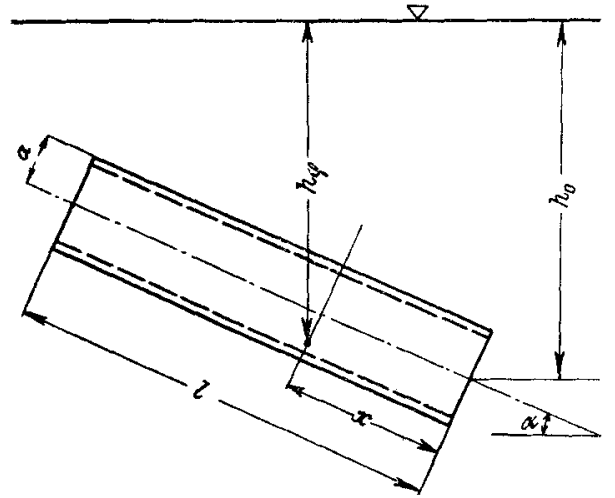

Abb. 3 ungeändert bleiben, da nur die in denselben auftretenden Konstanten $C_{1} \ldots C_{4}$ durch $C_{1 g} \ldots C_{g_{g}}$ zu ersetzen sind.

III. Das geneigt liegende Rohr.

1. Spannungen infolge des Flüssigkeitsgewichts. Nachdem im vorstehenden die Spannungen infolge des Flüssigkeits- und Eigengewichts für das wagerecht liegende Rohr ermittelt worden sind, soll nunmehr angenommen werden, das die Rohrachse unter dem Winkel $\alpha$ gegen die Horizontale geneigt liegt. In diesem Falle beträgt nach Abb. 3 der Flüssigkeitsdruck für die Flächeneinheit in der jetzt schräg gemessenen Entfernung $x$ vom unteren Rohrende, an dem - auf der Achse - die Druckhöhe $h_{0}$ vorbanden sei,

$$
p=\gamma h_{\varphi}=\gamma\left[h_{0}-x \sin \alpha+a \cos \varphi \cos \alpha\right]=\gamma\left[h_{0}-h_{1} \xi+a \cos \varphi \cos \alpha\right],
$$

wenn $h_{1}=a \sin \alpha$ die Druckhöhenänderung in der Rohrachse auf eine schräge Länge gleich dem Rohrradius darstellt. Demnach lauten dann die Gleichgewichtsbedingangen: $\left.\begin{array}{lr}T_{1}^{\prime}-S_{2}{ }^{\prime}=0 & H_{1}{ }^{\prime}-G_{3}{ }^{\prime}+N_{3} a=0 \\ S_{1}{ }^{\prime}+T_{2}+N_{2}=0 & G_{1}{ }^{\prime}+H_{2} \cdot-N_{1} a=0 \\ N_{1}{ }^{\prime}+N_{2} \cdot-T_{2}+a \gamma\left(h_{0}-h_{1} \xi+a \cos \alpha \cos q\right)=0 & -H_{2}+\left(S_{1}+S_{2}\right) a=0\end{array}\right\}$ $(\mathrm{III} \mathbf{g})$, whbrend die Formänderungsbedingungenen (I) (II) ungeändert bleiben. 
Man erkennt nun aus der Form des Störungsgliedes in (IIIg), daß die Gesamtspannungen sich auch hier wieder aus einem rotationssymmetrischen und einem sinusbezw. cosinusförmigen Spannungszustand additiv zusammensetzen werden.

Bezüglich des letzteren zeigt der Vergleich des ihm entsprechenden Störungsgliedes $a^{2} \gamma \cos \alpha$ mit dem des bereits unter 2 bis 4 untersuchten Spannungezustandes $a^{2} \gamma$, daß die gesuchten Spannungen durch genau die gleichen Formeln wie oben sich bestimmen, wofern nur in denselben überall $\gamma \cos \alpha$ statt $\gamma$ gesetzt wird; eine besondere Betrachtung erübrigt sich daher. Für die rotationssymmetrisch verteilten Spannungen hingegen lauten jetzt die Gleichgewichts- und Formänderungsbedingungen:

$$
\begin{aligned}
& T_{1}^{\prime}=0 \text { somit: } T_{1}=c, \quad N_{1}^{\prime}-T_{2}+a \gamma\left(h_{0}-h_{1} \xi\right)=0, \\
& a \varepsilon_{1}=u^{\prime}, \quad a \varepsilon_{2}=u, \quad a^{2} \varkappa_{1}=u^{\prime \prime}, \\
& T_{1}=\frac{12 D}{d^{2}}\left(\varepsilon_{1}+\sigma \varepsilon_{2}\right)=c=c \\
& T_{2}={ }_{d^{2}}^{12 D}\left(\varepsilon_{z}+\sigma \varepsilon_{1}\right) \\
& G_{1}=-D x_{1}+T_{1} a \varepsilon=-D x_{1}+c a \varepsilon \\
& G_{i}=-D \sigma x_{1}
\end{aligned}
$$

Eliminiert man wieder zunachst $N_{1}$ aus (IIIh), so folgt

$$
G_{1}^{\prime \prime}-a T_{2}+a^{2} \gamma\left(h_{0}-h_{1} \xi\right)=0
$$

und mit $T_{1}=c$, wenn in den aus (IIh) folgenden Beziehungen $\varepsilon_{2}{ }^{\prime}=a x_{1} ; x_{2}=0$ mittels ([h) Dehnnngen und Krümmungsänderungen durch die Spannungsresultanten ausgedrückt werden :

mithin wie S. $347^{\prime}$ ):

$$
\varepsilon_{d}^{\prime \prime}=\frac{T_{2}{ }^{\prime \prime}-T_{1}^{\prime \prime}:}{\left.E^{\prime}\right)^{\prime}}:=\frac{G_{1}(\mathrm{IV})}{E^{\prime}{ }_{a b}}=a x_{1}=\frac{a}{D}\left(-G_{1}+c a t\right),
$$

$$
G_{1}(\mathrm{IV})+\frac{12 a^{2}}{\delta^{2}}\left(1-\sigma^{3}\right) G_{1 \alpha}-c a\left(1-\sigma^{3}\right)=0
$$

Es bleibt also außer $T_{1}=c$ auch $G_{1}$ wie $S .347$ berechnet, nämlich: $G_{1 \alpha}=C_{i \alpha} \sin (\mu \xi) \operatorname{Sin}(\mu \xi)+C_{2 \alpha} \sin (\mu \xi) \sin (\mu \xi)+C_{4 \alpha} \cos (\mu \xi)$

wo

$$
+C_{s} \alpha \cos (\mu \xi)(50 j(\mu \xi) \sin (\mu \xi)+c a \varepsilon,
$$

$$
\mu=\frac{\prime}{\sqrt{2}} \quad \nu^{4}=\frac{13 n^{2}}{\delta^{2}}\left(1-\sigma^{2}\right) \text {. }
$$

Hingegen ergibt sich jetzt für $T_{2}$ :

$$
\begin{aligned}
T_{2 \alpha}=\frac{G_{1}^{\prime \prime}}{a}+a \gamma\left(h_{0}-h_{1}\right) \xi= & \frac{\nu^{2}}{a}\left[-C_{4 \alpha} \sin (\mu \xi) \operatorname{Sin}(\mu \xi)-C_{3 \alpha} \sin (\mu \xi)(\operatorname{Soj}(\mu \xi)\right. \\
& +C_{2 \alpha} \cos (\mu \xi) \operatorname{Sin}(\mu \xi)+C_{3 \alpha} \cos (\mu \xi)(\operatorname{Goj}(\mu \xi)]+a \gamma\left(h_{0}-h_{1} \xi\right),
\end{aligned}
$$

ferner für $G_{2}$ und $N_{1}$ wie S. 347 :

$$
\begin{aligned}
& G_{2 \alpha}=\sigma G_{1}-\sigma c a \varepsilon=\sigma\left[C _ { 1 \alpha } \operatorname { s i n } \left(\mu \xi ; \operatorname{Sin}(\mu \xi)-C_{2 \alpha} \sin (\mu \xi)\left(S_{0} \mid(\mu \xi)\right.\right.\right. \\
& +C_{3 x} \cos (\mu \xi) \operatorname{Sin}(\mu \xi)+C_{4 x} \cos (\mu \xi)(50 j(\mu \xi)] \\
& N_{1 \alpha}=\frac{v^{\prime}}{a V^{2}}\left[+\begin{array}{l}
\left(C_{2 \alpha}-C_{3 \alpha}\right) \sin (\mu \xi) \operatorname{Sin}(\mu \xi)+\left(C_{1 \alpha}-C_{4 \alpha}\right) \sin (1 t \xi)\left(S_{0}(\mu \xi)\right. \\
\left(C_{1 \alpha}+C_{4 \alpha}\right) \cos (\mu \xi) \operatorname{Sin}(\mu \xi)+\left(C_{2 \alpha}+C_{3 \alpha}\right) \cos (\mu \xi)\left(S_{0 j}(\mu \xi)\right.
\end{array}\right],
\end{aligned}
$$

während sich für die Verschiebnngen ergibt:

$$
\begin{aligned}
& w_{\alpha}=a \varepsilon_{2 \alpha}={ }_{E}^{a} \delta T_{2 \alpha}-\sigma T_{1 \alpha} \\
& u_{\alpha}=a \int \varepsilon_{1} d \xi={ }_{E \varrho Q}^{a} \int\left(T_{1 \alpha}-T_{2 \alpha} \sigma\right) d \xi=\frac{a}{E \delta} \int\left\{c_{\alpha}-\sigma\left[N_{1} a^{\prime}+a \gamma\left(h_{0}-h_{1} \xi\right)\right]\right\} d \xi
\end{aligned}
$$

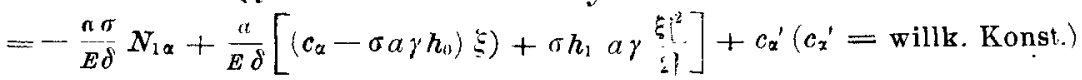

und für die Tangentendrehung:

$$
\begin{aligned}
& w_{\alpha}^{\prime}=\frac{a}{E \delta} T_{2 \alpha^{\prime}}=\frac{\nu^{\prime 3}}{E \delta V_{2}}\left\{-\left(C_{2 \alpha}+C_{3 \alpha}\right) \sin (\mu \xi) \operatorname{Sin}\left(\mu_{\xi}^{\prime}\right)-\left(C_{1 \alpha}+C_{1 \alpha}\right) \sin (\mu \xi)\left(C_{0} j(\mu \xi)\right.\right. \\
& +\left(C_{1 \alpha}-C_{4 \alpha}\right) \cos (\mu \xi) \operatorname{Sin}\left(\mu^{\alpha} \xi\right)+\left(C_{2 \alpha}-C_{3 \alpha}\right) \cos (\mu \xi)\left(\mathcal{S} 0 \eta(\mu \xi)-a \gamma h_{1} .\right.
\end{aligned}
$$

2. Spannungen infolge des Rohreiget,rgewichts. In ebenso übersichtlicher Weise wie für das Flüssigkeitsgewicht lassen ouch auch für das Rohreigengewicht die Spannungen im schräg liegenden Rohr auf die dobs horizontalen Rohrs zurïckfïhren.

') Der Index $\alpha$ kennzelchnet Spannungs. und Defor 'x ationsgrößen des unter $a$ geneigten Rohres. 
Für das letztere lauten nämlich, wie man leicht sieht, die Gleichgewichtsbedingungen bei Beanspruchnog allein durch Rohreigengewicht:

$$
\begin{array}{ll}
T_{1}^{\prime}-S_{2}{ }^{\circ}-a^{2} \gamma^{\prime} E^{\prime} \sin \alpha=0 & H_{1}^{\prime}-G_{2}{ }^{\circ}+N_{2} a=0 \\
S_{1}^{\prime}+T_{2}^{\prime}+N_{2}-a^{2} \gamma_{E^{\prime}} \cos \alpha \sin q=0 & G_{1}^{\prime}+H_{2} \cdot N_{1} a=0 \\
N_{1}^{\prime}+N_{2} \cdot T_{2}+a^{2} \gamma_{E^{\prime}} \cos \alpha \cos \varphi=0 & -H_{2}+\left(S_{1}+S_{2}\right) a=0
\end{array}
$$

Vergleicht man diese mit den oben angegebenen Gleichungen für das horizontale Rohr, so erkennt man, daß dieselben sich, wenn man von dem Störungsglied $-a^{2} \gamma_{E}^{\prime}$ $\sin \alpha$ der $X$-Richtung zunächst absieht, nur um den konstanten Faktor $\cos \alpha$ in den beiden Störungsggliedern der $Y$ - und $Z$-Richtung unterscheiden. Hieraus geht hervor, daß die Gesamtspannungen sich wieder aus einem sinus- bezw. cosinueförmigen Spannungszustand zusammensetzen, der sich einfach aus den früheren Werten dadurch ergibt, das überall $\gamma_{F^{\prime}}^{\prime} \cos \alpha$ statt $\gamma_{E}^{\prime}$ geschrieben wird, sowie einem rotationssymmetrischen Zustand für den die Gleichgewichtsbedingangen lauten:

$$
T_{1}^{\prime}=a^{2} \gamma_{E^{\prime}}^{\prime} \sin \alpha ; \quad \text { mithin: } T_{1}=a^{2} \gamma^{\prime} k^{\prime} \xi \sin \alpha+c_{a}^{*}
$$

ferner:

$$
N_{1}{ }^{\prime}-T_{2}=0, \quad G_{1}{ }^{\prime}-N_{1} a=0 \text {. . . . . . . (IIIi), }
$$

$$
\begin{aligned}
& a \varepsilon_{1}=u^{\prime} \quad a \varepsilon_{2}=w \quad a^{2} x_{1}=w^{\prime \prime} \quad x_{2}=0 \\
& \left.\left.\begin{array}{l}
T_{1}=-\frac{12 D}{\partial^{2}}\left(\varepsilon_{1}+\sigma \varepsilon_{2}\right) \\
T_{2}=\frac{12 D}{\delta^{\prime}}\left(\varepsilon_{2}+\sigma \varepsilon_{1}\right)
\end{array}\right\} \quad \cdot \quad \text { (II i) } \quad \begin{array}{l}
G_{1}=-D \varkappa_{1}+T_{1} a \varepsilon \\
G=-D \sigma \varkappa_{1}
\end{array}\right\}
\end{aligned}
$$

Die Elimination von $N_{1}$ aus (III i) ergibt:

$$
G_{1}^{\prime \prime}-a T_{2}=0,
$$

hierzu mit $T_{1}=\xi a^{2} \gamma^{\prime}{ }^{\prime} \sin \alpha+c_{a}^{3}$ und $\varepsilon_{2}^{\prime \prime}=a x_{1}: x_{3}=0$, mit Benutzung yon (Ij):

$$
\varepsilon_{2}^{\prime \prime}=\frac{T_{2}{ }^{\prime \prime}-T_{1}^{\prime \prime} \sigma}{E \delta}=\frac{G_{1}(\mathrm{IV})}{E \delta a}=a x_{1}=\stackrel{a}{D}_{D}^{a}\left|-G_{1}+\iota \varepsilon\left(c_{a}^{*}+\xi a^{2} \gamma_{E^{\prime}} \sin \alpha\right)\right| .
$$

Hieraus:

$$
G_{1}(\mathrm{IV})+\frac{12 a^{2}}{\partial^{2}}\left(1-\sigma^{2}\right) G_{1}-a\left(1-\sigma^{2}\right)\left(c_{\alpha}^{*}+5 a^{2} \gamma_{E^{\prime}} \sin \alpha\right)=0 .
$$

Das Integral lautet:

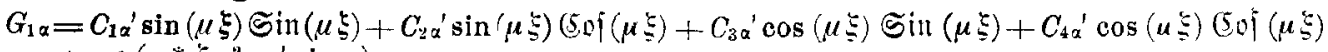
$+a \varepsilon\left(c_{\alpha}^{*} \xi a^{3} \gamma_{E}^{\prime} \sin \alpha\right)$.

Für die übrigeu Spannungen und Formänderungen findet man:

$T_{2 \alpha}=\frac{G_{1}^{\prime \prime}}{a}=\frac{v^{2}}{a}\left\lfloor-C_{4 \alpha^{\prime}} \sin (\mu \xi) \operatorname{Sin}(\mu \xi)-C_{3 \alpha^{\prime}} \sin (\mu \xi) \circlearrowleft D(\mu \xi)+C_{2} \alpha^{\prime} \cos (\mu \xi) \sin (\mu \xi)\right.$

$G_{2 \alpha}=\sigma G_{1 \alpha}-\sigma a \varepsilon T_{1 \alpha}=\mid C_{1 \alpha} \sin (\mu \xi) \operatorname{Sin}(\mu \xi)+C_{2 \alpha} \sin (\mu \xi)(\delta)(u \xi)$

$+C_{1} \omega^{\prime} \cos (\mu \xi)(50 j(u \xi)]$. $+C_{3 \alpha}{ }^{\prime} \cos (\mu \xi) \operatorname{Sin}(\mu \xi)+C_{4 \alpha^{\prime}} \cos (\mu \xi) \operatorname{Coj}(\mu \xi)$

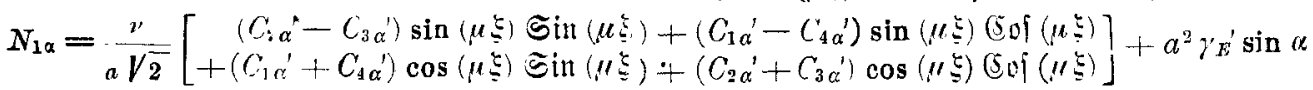

$$
\begin{aligned}
& u \alpha=a \varepsilon_{2}=\frac{a}{E \delta}\left(T_{a \alpha}-\sigma T_{1_{\alpha}}\right) \\
& u_{c}=a \int \varepsilon_{1} d \xi=\frac{a}{E \delta} \int\left(T_{1 \alpha}-T_{2 \alpha} \sigma\right) d \xi \xi=\frac{a}{E} \delta \int\left\{\xi a^{2} \gamma_{H^{\prime}} \sin \alpha+c \alpha^{*}-\sigma N_{1 \alpha}\right\} d \xi \\
& =-{ }_{E \delta}^{a \delta} N_{1 !}+\frac{a}{E \delta}\left(c a^{*} \xi+\frac{\xi^{2}}{2} a^{2} \gamma_{Z^{\prime}} \sin a\right)+c_{e^{* \prime}}\left(c_{t^{*}}^{* \prime}=\text { willk. Konst. }\right)
\end{aligned}
$$

$w_{\alpha}^{\prime}=\frac{a}{E \delta}\left(T_{2 \alpha^{\prime}}-T_{1 \alpha^{\prime}}\right)$

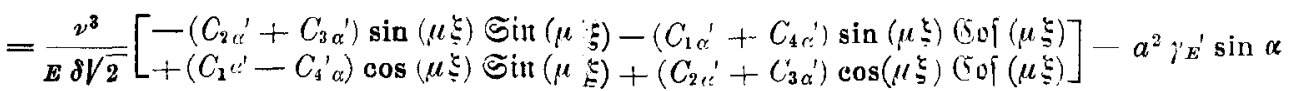

Dieser Spannungozustand ist de,m in II, 2, $c$ gegebenen (mit $\gamma_{E}^{\prime} \cos \alpha$ stalt $\gamma_{E^{\prime}}$ ) zu überlagern, womit dann die Berechnun $g$ des schräg liegenden Rohres vollständig auf die des horizontalen zurückgeführt ist. 


\section{Zusammenfassung.}

Die in der vorliegenden Arbeit gegebenen theoretischen Grundlagen ermöglichen es, die in schrägen oder horizontalen freitragenden Rohrleitnngen beliebiger Stïtzung - insbesondere auch Walzenkesseln - auftretenden Spannungen genauer als bisher zu berechnen und insbesondere sioh über die beträchtlichen Spannungen in der Nähe der Auflager ein der Wirklichkeit năher kommendes Bild zu machen.

Die für dü̈nwandige zylindrische biegungefeste Schalen gültigen Gleichgewichtsund Formänderungsbedingungen werden zunächst für den Fall des horizontalen, durch Flüssigkeitsdruck beanspruchten Rohres aufgestellt und in geschlossener Form vollständig integriert, wobei in der Weise vorgegangen wird, daß der Einfluß der einzelnen Störungsglieder getrent verfolgt wird.

Hierbei ergibt sich, daß die im Rohr auftretenden Gesamtspannungen sich aus der Ueberlagerung zweier Spannungszustände zusammensetzen, von denen der eine inbezug auf die Rohrachse symmetrisch, der andere sinus- bezw. cosinusförmig ver teilte Spannungen hervorrult.

Die fïr jeden dieser Spannungszustände getrennt anzusetzenden Randbedingungen werden für alle praktisch wichtigen Stützungsarten, insbesondere anch für kontinuierliche Leitungsstränge angegeben.

Nachdem sodann auch der Einflus des Rohreigengewichts für das horizontale Rohr vollstăndig ermittelt ist, wird das biegungsfeste, geneigt liegende Rohr gleichfalls auf Flïssigkeits- und Eigergewiohtsbelastung untersucht und gezeigt, daß sioh bei wiederum getrennter Verfolgung des Einflusses der einzelnen Störungsglieder die Spannungen in geneigt liegenden Rohren in sehr einfacher und übersichtlicher Weise auf die im horizontalen Rohr auftretenden zurückführen lassen.

\section{Über die Wurzeln der charakteristischen Gleichungen von Schwingungsproblemen.}

\section{Von GEORG PICK in Prag. 1 )}

D' ie Gleịchungev, welche entstehen, wenn wan von den Diagonalelementen einer gegebenen quadratisehen Matrix die Unbekannte $t$ subtrahiert, und die erhaltene Determinante gleich Null setzt, haben lauter raelle Wurzeln in dem Falle, daß die gegebene Matrix symmetrisch ist. Es liegt deshalb nahe, für den allgemeinen Fall eine Abschätzung des Imaginärteils der Wurzel zu suchen, der diesen Teil in Abbängigkeit setzt von dor Abweichung der Matrix von der Symmetrie. Von diesem Gedanken ausgehend hat Bendix son solohe Gleichungen untersucht, und Hirsch hat die Untersuchung auf Matrizen mit komplexen Elementen ausgedehnt $\left.{ }^{2}\right)$.

Wenn diese Frage nun hier zunächst unter Beschränkung auf reelle Matrizen wieder anfgenemmen wird, so geschieht das, weil die von Bendixson erzielte Abschätzung des Imaginärteils erheblich verbessert, nämlich durch eine s charfo Abschätzung erselzt werden kann. Sind $a_{\mu \nu}$ die Elemente der gegebenen $n$-zeiligen Matrix, und bezeichnet man den größten unter den Werten

$$
\left|\frac{a \mu y-a y \mu}{2}\right|
$$

mit $g$, so findet Bendixson für den Tmaginärteil der Wurzel $t=r+s i$ die Beziehung

$$
-g \sqrt{\frac{n(n-1)}{2}} \leq s \leqq+g \sqrt{\frac{n(n-1)}{2}} .
$$

1) Die gewöhnlichen Schwingungsproblewe der Mechanik fuhren aup ein System von linearen Gilichungen, die eine etwas allgemeinere Form haben, als die vom Verfasser hier vorausgesetzten. Da man nämlich stets Differentialgleichungen zweiter Ordnung hat, tritt der Exponent $\lambda$ nicht linear, sondern quadratisch in dem Koefizientenschema (Matrix) der linearen Gleichungen auf. Eine unmittelbare Anwendung der Ergebnisse der nacbstehenden Arbeit auf die Entscheidung der Stabilitatsfragc bei deralticen Sehwingungsproblemen wird daher kaum möglich sein. Wohl aber kommen hier andere als mechanische Schwingungsvorgänge in Frage, vor allem Teinperaturschwankungen und elektrische Schwingungen, bei denen der EinfluB der Selbstinduktion vernachläsigt wird. (Anmerk ung des Herausgebers.)

2) Bendixson, Acta mathematica XXV, S. 359-365, Hirsch, ehenda S. 367-370. 